\title{
Systolic Blood Pressure Response to Exercise as a Predictor of Mortality in Patients With Chronic Heart Failure
}

\author{
Yasuhiro NishiYama, ${ }^{1}$ MD, Hirohiko Morita, ${ }^{1}$ MD, Haruhito Harada, ${ }^{1}$ MD, Atsushi Katoh, ${ }^{1}$ MD, \\ Hisashi AdachI, ${ }^{2} \mathrm{MD}$, Yoshinori KogA, ${ }^{1} \mathrm{MD}$, and Hisao IKedA, ${ }^{1} \mathrm{MD}$
}

\section{SUMMARY}

It is well known that peak oxygen consumption and heart rate (HR) recovery after exercise obtained from the cardiopulmonary exercise test are prognostic parameters in patients with chronic heart failure (CHF). However, it is unclear whether exercise-induced parameters obtained from the routine exercise stress test predict mortality in patients with CHF.

We studied 136 patients (93 males/43 females) with CHF. All patients underwent symptom-limited exercise stress testing. Exercise parameters included exercise duration, exercise-induced HR and systolic blood pressure (SBP), and metabolic equivalents (METs).

During the follow-up period (mean 6.2 years), 34 patients died. Survival rates at the 3 rd and 5 th years were $90 \%$ and $83 \%$, respectively. Body mass index was significantly smaller in the nonsurvival group than in the survival group $(P<0.01)$. The incidence of patients with New York Heart Association III class was higher in the nonsurvival group than in the survival group $(P<0.05)$. In univariate analysis, predictors of mortality included peak HR and SBP, increases in HR and SBP during exercise, HR and SBP at the 1st minute after exercise, HR at the 3rd minute after exercise, and METs. The use of $\beta$-adrenergic blocking agents was not associated with prognosis. In Cox hazard model analysis, the increase in SBP $(P<0.002)$, HR at the 3rd minute after exercise $(P<0.05)$, and METs $(P<0.05)$ were independent predictors of mortality.

SBP response to exercise, HR recovery after exercise, and METs obtained from the routine exercise test predicted mortality in patients with CHF irrespective of the use of $\beta$-adrenergic blocking agents. (Int Heart J 2010; 51: 111-115)

Key words: Exercise stress test, Heart failure, Heart rate, Systolic blood pressure, $\beta$-adrenergic blocking agents

$\mathrm{C}$ hronic heart failure (CHF) is a major cause of morbidity and mortality, and its prevalence is increasing in many countries. ${ }^{1)}$ Hence, it is of importance to provide clinical prognostic information for improving patient care. Several variables obtained from cardiopulmonary exercise testing have demonstrated their prognostic value in patients with CHF. Of these, peak oxygen consumption $\left(\mathrm{VO}_{2}\right)$ is a strong predictor to risk-stratify patients with $\mathrm{CHF}^{2)}$ Indeed, peak $\mathrm{VO}_{2}$ is most likely used in selecting patients for cardiac transplantation. ${ }^{2)}$ The minute ventilation/carbon dioxide production slope by ventilatory gas analysis was also shown to be useful as a prognostic parameter in patients with $\mathrm{CHF}^{3,4)}$ Moreover, several other cardiopulmonary exercise variables, including heart rate (HR) recovery after exercise, have recently been shown to be impaired in patients with CHF, indicating they are significant predictors of cardiovascular mortality. ${ }^{5-7)}$ Thus, these studies were performed with ventilatory gas analysis in patients with $\mathrm{CHF}^{8-12)}$ However, cardiopulmonary exercise testing cannot be always performed as a routine exercise stress test in a clinical setting. Accordingly, it may be necessary to identify the prognostic value of the exercise stress test vari- ables in patients with CHF when ventilatory gas analysis is not available. Therefore, the aim of the present retrospective study was to investigate whether exercise parameters obtained from routine exercise stress testing predict mortality in patients with CHF.

\section{MeTHODS}

Study patients: Patients with CHF who were admitted to Kurume University Medical Center in July 1994 to March 2004 and fulfilled the following criteria were enrolled as subjects. The criteria for entry were: New York Heart Association (NYHA) functional class II or III and performance of exercise stress test, and follow-up duration $>1$ year. The endpoint of all cause mortality was assessed by a standardized questionnaire. Consequently, 136 patients with CHF were diagnosed based on their physical condition such as pulmonary congestion or peripheral edema, standard 12lead electrocardiography (ECG), chest x-ray, and echocardiography. Organic heart diseases causing heart failure were dilated cardiomyopathy in 30, valvular heart disease in 27 ,

From the ${ }^{1}$ Department of Cardiology, Kurume University Medical Center, and ${ }^{2}$ Department of Cardio-Vascular Medicine, Kurume University School of Medicine, Fukuoka, Japan.

Address for correspondence: Yasuhiro Nishiyama, MD, Nishiyama Clinic, 115-4 Waifu, Kikuchi, Kumamoto 861-1331, Japan.

Received for publication September 30, 2009.

Revised and accepted January 7, 2010. 
old myocardial infarction in 22 , hypertensive heart disease in 20, hypertrophic cardiomyopathy in 18 , coronary artery disease in 7, arrhythmia in 5, and others in 7. Patients with atrioventricular block and pacemaker implantation were excluded from the study. All patients received conventional drug therapy. In these patients, medications included diuretics $(n=89)$, angiotensin converting enzyme inhibitors or angiotensin receptor blockers $(n=80)$, isosorbide dinitrate $(n=63)$, calcium channel antagonists $(n=61)$, digitalis $(n=56)$, antiarrhythmic agents $(n=52), \beta$-adrenergic blocking agents $(n=36)$, nicorandil $(n=21)$, dopamine agents $(n=8), \alpha$-adrenergic blocking agents $(n=5)$, and others $(n=2)$. Informed consent was obtained from all patients, and the study was approved by the Human Study Committee of our institution.

Exercise stress test: All patients underwent a symptom-limited exercise stress test. The treadmill exercise stress tests (ML4500, Fukuda Denshi, Tokyo) were performed using the Bruce (low level) or Sheffield protocol. The ECG and systolic blood pressure (SBP) were recorded every minute before, during, and after exercise. Criteria for stopping the exercise test included chest discomfort, life-threatening arrhythmias, ST-segment depression or elevation $>0.2 \mathrm{mV}$, a fall in SBP from the preceding stage $>20 \mathrm{mmHg}$, or predicted target HR [(220 - age $) \times(0.85)]$. Exercise parameters included exercise duration, HR and SBP at rest, peak exercise, 1 st or 3 rd minute after exercise, and metabolic equivalents (METs).

Statistical analysis: Values are presented as the mean \pm $\mathrm{SD}$. The comparisons between groups were made by the unpaired $t$-test and $\chi^{2}$ test. The survival curve was calculated by the Kaplan-Meier method with log-rank testing. The cut-off values for the exercise parameters were based on the mean value of data obtained from the present study. Cox proportional hazard stepwise analysis was performed for determinants of the survival rate adjusted for age and sex. All statistical analyses were performed using the SPSS system. A probability value of $<0.05$ was considered to be significant.

\section{RESUlts}

Baseline characteristics: The baseline characteristics of the study patients are shown in Table I. There were no differences in age, sex, etiology of heart failure, ejection fraction, or the use of $\beta$-adrenergic blocking agents between the survival and nonsurvival groups. Body mass index was significantly lower in the nonsurvival group than that in the survival group $\left(21.0 \pm 2.9\right.$ versus $22.8 \pm 3.3 \mathrm{~kg} / \mathrm{m}^{2}, P<$ $0.01)$. Patients with NYHA III were significantly greater in the nonsurvival group than in the survival group (64.7 versus $38.3 \%, P<0.05)$. Moreover, plasma brain natriuretic peptide levels were significantly higher in the nonsurvival group than those in the survival group $(553 \pm 505$ versus $208 \pm 250 \mathrm{pg} / \mathrm{mL}, P<0.0001)$.

Clinical course: One hundred and thirty-six patients (93 males and 43 females) responded to the follow-up interview. During the mean follow-up period of $6.2 \pm 2.5$ years, 34 patients died $(25 \%)$. In the survival curve (Figure 1), survival rates at the 3 rd and 5th year were $90 \%$ and $83 \%$,

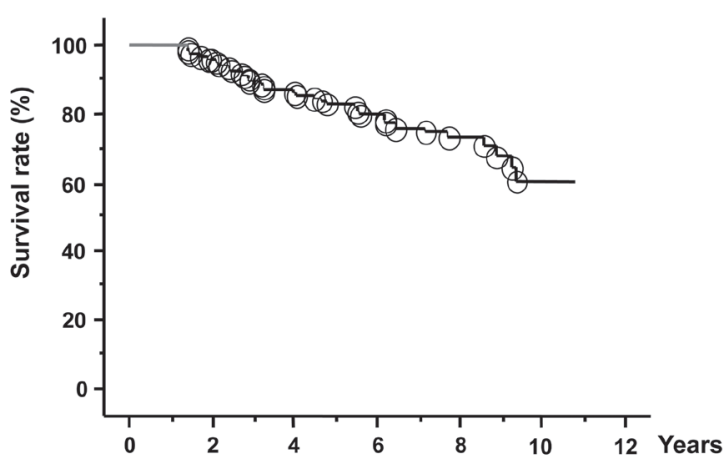

Figure 1. Kaplan-Meier plot relating survival to mortality.

Table I. Baseline Characteristics

\begin{tabular}{lcc}
\hline & $\begin{array}{c}\text { Survival } \\
(n=102)\end{array}$ & $\begin{array}{c}\text { Nonsurvival } \\
(n=34)\end{array}$ \\
\hline Age & $63 \pm 12$ & $61 \pm 13$ \\
Sex $($ male/female) & $78 / 24$ & $25 / 9$ \\
BMI $\left(\mathrm{kg} / \mathrm{m}^{2}\right)$ & $22.8 \pm 3.3$ & $21.0 \pm 2.9^{* *}$ \\
Etiology $(\%)$ & & \\
CAD & $20(19.6)$ & $9(26.4)$ \\
Hypertension & $16(15.6)$ & $4(11.7)$ \\
DCM & $22(21.6)$ & $8(23.5)$ \\
HCM & $10(9.8)$ & $8(23.5)$ \\
Valvular & $24(23.5)$ & $3(8.8)$ \\
NYHA $(\%)$ & & $12(35.3)$ \\
$\quad$ II & $63(61.7)$ & $22(64.7)^{*}$ \\
$\quad$ III & $39(38.3)$ & $8(23.5)$ \\
$\beta$-Adrenergic blocking agents $(\%)$ & $28(27.5)$ & $46 \pm 19$ \\
Ejection fraction $(\%)$ & $50 \pm 16$ & $553 \pm 505^{* * *}$ \\
BNP (pg/mL) & $208 \pm 250$ & \\
\hline
\end{tabular}

${ }^{*} P<0.05,{ }^{* *} P<0.01$ and ${ }^{* * *} P<0.0001$ as compared to survival group. $\mathrm{BNP}$ indicates brain natriuretic peptide; $\mathrm{CAD}$, coronary artery disease; DCM, dilated cardiomyopathy; HCM, hypertrophic cardiomyopathy; and, NYHA, New York Heart Association.

Table II. Exercise Parameters of Survival and Nonsurvival Groups

\begin{tabular}{lrc}
\hline Variable & $\begin{array}{r}\text { Survival } \\
(n=102)\end{array}$ & $\begin{array}{c}\text { Nonsurvival } \\
(n=34)\end{array}$ \\
\hline Heart rate (beats/minute) & $77 \pm 14$ & $73 \pm 9$ \\
$\quad$ Rest & $127 \pm 23$ & $116 \pm 21^{*}$ \\
Peak exercise & $115 \pm 22$ & $105 \pm 20^{*}$ \\
First minute after exercise & $91 \pm 15$ & $84 \pm 15^{* *}$ \\
Third minute after exercise & $50 \pm 20$ & $43 \pm 18$ \\
$\Delta$ HR & $129 \pm 21$ & $127 \pm 20$ \\
Systolic BP (mmHg) & $171 \pm 34$ & $154 \pm 39^{*}$ \\
Rest & $160 \pm 31$ & $147 \pm 33^{*}$ \\
Peak exercise & $145 \pm 24$ & $141 \pm 23$ \\
First minute after exercise & $42 \pm 32$ & $27 \pm 37^{*}$ \\
Third minute after exercise & $5.0 \pm 3.6$ & $4.1 \pm 1.5^{*}$ \\
$\Delta$ BP & & \\
METs &
\end{tabular}

${ }^{*} P<0.05$ and ${ }^{* *} P<0.01$ as compared to survival group.

$\mathrm{HR}$ indicates heart rate; $\triangle \mathrm{HR}, \mathrm{HR}$ at peak exercise - HR at rest; SBP, systolic blood pressure; $\triangle \mathrm{SBP}$ at peak exercise - SBP at rest; and, METs, metabolic equivalents. 
Table III. Univariate Analysis

\begin{tabular}{lccc}
\hline Variable & $\beta$ & SE & $P$ \\
\hline Age & 0.001 & 0.014 & 0.997 \\
Sex & 0.167 & 0.391 & 0.669 \\
Ischemic & 0.237 & 0.369 & 0.544 \\
Heart rate (beats/minute) & & & \\
$\quad$ Rest & -0.016 & 0.013 & 0.235 \\
$\quad$ Peak exercise & -0.019 & 0.008 & $0.012^{*}$ \\
$\quad$ First minute after exercise & -0.020 & 0.006 & $0.020^{*}$ \\
$\quad$ Third minute after exercise & -0.032 & 0.012 & $0.008^{* *}$ \\
$\quad \Delta$ HR & -0.020 & 0.009 & $0.037^{*}$ \\
Systolic Blood Pressure (mmHg) & & & \\
$\quad$ Rest & -0.004 & 0.009 & 0.673 \\
$\quad$ Peak exercise & -0.014 & 0.006 & $0.014^{*}$ \\
$\quad$ First minute after exercise & -0.013 & 0.006 & $0.027^{*}$ \\
$\quad$ Third minute after exercise & -0.004 & 0.008 & 0.569 \\
$\quad \Delta$ SBP & -0.013 & 0.006 & $0.017^{*}$ \\
METs & -0.203 & 0.093 & $0.029^{*}$ \\
Atrial fibrillation & -0.165 & 0.454 & 0.716 \\
$\beta$-Adrenergic blocking agents & -0.179 & 0.405 & 0.658 \\
\hline
\end{tabular}

${ }^{*} P<0.05$ and ${ }^{* *} P<0.01$ as compared to survival group. Abbreviations as shown in Table II.

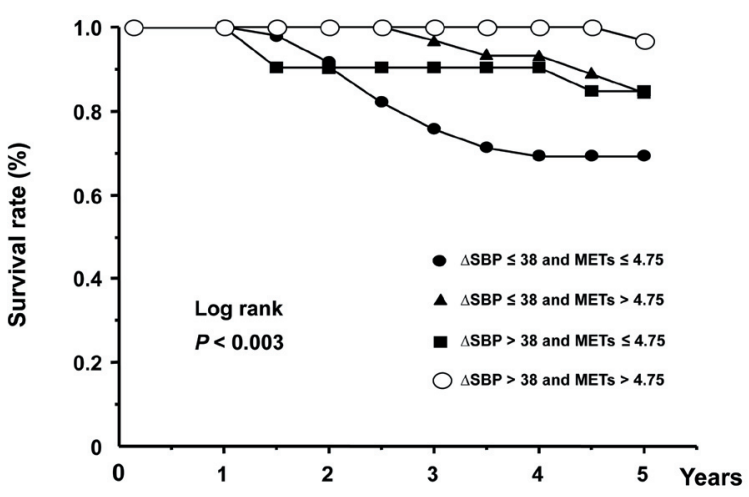

Figure 2. Kaplan-Meier plot relating survival to the combination of an increase in $\mathrm{SBP}(\triangle \mathrm{SBP})$ with metabolic equivalents (METs).

Table IV. Cox Proportional Hazard Stepwise Regression Analysis

\begin{tabular}{lcccc}
\hline Variable & $\beta$ & SE & $P$ & $\begin{array}{c}\text { Hazard ratio } \\
\text { (95\% confidence intervals) }\end{array}$ \\
\hline$\Delta$ SBP & -0.017 & 0.006 & 0.002 & $0.983(0.972-0.994)$ \\
HR at the third minute after exercise & -0.026 & 0.012 & 0.033 & $0.974(0.951-0.998)$ \\
METs & -0.228 & 0.002 & 0.038 & $0.998(0.990-0.998)$ \\
Peak systolic blood pressure & -0.011 & 0.009 & 0.243 & $0.989(0.972-1.007)$ \\
$\Delta$ HR & -0.010 & 0.010 & 0.288 & $0.990(0.972-1.009)$ \\
SBP at the first minute after exercise & -0.005 & 0.007 & 0.462 & $0.995(0.981-1.009)$ \\
Peak heart rate & -0.007 & 0.010 & 0.513 & $0.993(0.974-1.013)$ \\
HR at the first minute after exercise & -0.004 & 0.014 & 0.751 & $0.969(0.969-1.023)$ \\
\hline
\end{tabular}

Abbreviations as shown in Table II.

respectively.

Exercise parameters: The exercise parameters for the survival and nonsurvival groups are shown in Table II. There were no differences between the groups in HR and SBP at rest, or the increase in $\mathrm{HR}$ and SBP at the 3rd minute after exercise. However, HR and SBP at peak exercise, $\mathrm{HR}$ at the 1st and 3rd minute after exercise, SBP at the 1st minute after exercise, and the increase in SBP and METs in the nonsurvival group were significantly lower than those in the survival group. Exercise parameters did not differ between patients with and without the use of $\beta$-adrenergic blocking agent. Furthermore, in the patients administered $\beta$-adrenergic blocking agents, exercise parameters including $\mathrm{HR}$ at peak exercise, the increases in HR and SBP were not different between the survival and nonsurvival groups.

Cox hazard model analysis: In univariate analysis (Table III), predictors of mortality included HR and SBP at peak exercise, the increase in HR and SBP during exercise, HR and SBP at the 1st minute after exercise, HR at the 3rd minute after exercise, and METs. Treatment with $\beta$-adrenergic blocking agents did not have an influence on prognosis. In Cox proportional hazard stepwise regression analysis (Table IV), the increase in SBP during exercise was the most independent predictor of mortality in patients with
CHF. Moreover, HR at the 3rd minute after exercise and METs were independent predictors of mortality in those with CHF.

Combination of risks and mortality: Figure 2 presents the Kaplan-Meier plot relating survival to the combination of increases in SBP $(\triangle \mathrm{SBP})$ and METs $(\triangle \mathrm{SBP} \leqq 38$ and METs $\leqq 4.75, n=47 ; \Delta \mathrm{SBP} \leqq 38$ and METs $>4.75, n=$ $30 ; \Delta \mathrm{SBP}>38$ and METs $\leqq 4.75, n=21 ; \Delta \mathrm{SBP}>38$ and METs $>4.75, n=38$ ). When evaluating the significance of any combination of abnormally low $\triangle \mathrm{SBP}$, low METs and low HR at the 3rd minute after exercise, abnormally low $\triangle \mathrm{SBP}$ combined with a low METs was significantly associated with the highest risk of mortality in patients with $\mathrm{CHF}$ $(P<0.005)$. Furthermore, the combination of abnormally low $\triangle \mathrm{SBP}$ and low HR at the 3rd minute after exercise also identified the group at high risk for mortality $(P<0.03)$ (data not shown).

\section{Discussion}

In the present study, we demonstrated that the SBP response to exercise, HR at the 3rd minute after exercise, and METs obtained from the routine exercise test were in- 
dependent predictors of mortality in patients with CHF. Of these parameters, an abnormally low SBP response to exercise was the most powerful predictor of mortality in those with CHF.

It is widely recognized that exercise-induced blood pressure reflects cardiac output during exercise. A previous study has demonstrated that cardiac output increased during exercise and decreased after exercise much more slowly as CHF worsened. ${ }^{13)}$ Therefore, it is thought that the SBP response to exercise reflects an increase in cardiac output during exercise. Importantly, a blunted SBP response to exercise is associated with a poorer prognosis. In the previous studies with cardiopulmonary exercise tests, the blood pressure response to submaximal exercise was a significant predictor of prognosis in $\mathrm{CHF}^{14,15)}$ Although there are several differences in patient selection and methodology, our findings are consistent with previous results ${ }^{14,15)}$ showing that an abnormally low SBP response during exercise is a significant predictor of prognosis with $\mathrm{CHF}$.

In the previous studies, the HR response at the 1st, 2nd, or 3rd minute after exercise was shown to be a significant predictor of prognosis in patients with CHF. ${ }^{10-12)}$ Lipinski, et al demonstrated that HR recovery (maximum $\mathrm{HR}-\mathrm{HR}$ at specified time period during recovery) at the 2nd minute after exercise was the strongest predictor of mortality as compared to HR recovery at 3 or 5 minutes after exercise for $\mathrm{CHF}$ patients without $\beta$-adrenergic blocking agents. ${ }^{9)}$ In this study, HR at the 3rd minute after exercise was an independent predictor of mortality in CHF. Although this discrepancy may be due to the differences in study patients and methodology, it seems that HR recovery at a few minutes after exercise provides clinically important information for the prognosis of CHF patients. In general, an imbalance of the autonomic nervous system is recognized as a chronotropic incompetence response to exercise in CHF patients. ${ }^{6,16)}$ A previous study by Imai, et al showed that HR decay at the first 30 seconds after exercise was a specific vagally-mediated indicator while HR decay at the 2nd minute after exercise was not a specific vagally-mediated indicator. ${ }^{16)}$ This is because HR decay at the 2 nd minute after exercise was dependent on exercise intensity and sympathetic nerve dependence. Therefore, HR at the $3 \mathrm{rd}$ minute after exercise obtained from our study may reflect the complex condition of combined vagal and sympathetic nerve activation. Medication with $\beta$-adrenergic blocking agents depresses HR via sympathetic nerves. In this study, $26 \%$ of the patients took $\beta$-adrenergic blocking agents. This may have produced the difference in HR recovery time as compared to the study results reported by Lipinski, et $a l .{ }^{9)}$ In this study, no exercise parameters differed between the survival and nonsurvival groups in patients using an $\beta$-adrenergic blocking agent, although these findings may be attributable to the small number of patients and/or the dosage of $\beta$-adrenergic blocking agents. Moreover, the use of $\beta$-adrenergic blocking agents was not a prognostic predictor in CHF patients in this study. This finding may support the results of previous studies showing that the use of $\beta$-adrenergic blocking agents is not a useful predictor of prognosis in patients with $\mathrm{CHF}^{2,7)}$

Peak $\mathrm{VO}_{2}$ as a marker of exercise capacity is a strong predictor of mortality in patients with $\mathrm{CHF}^{2)}$ We used
METs as a marker of exercise capacity instead of peak $\mathrm{VO}_{2}$ because we could not measure peak $\mathrm{VO}_{2}$. In the present study, METs was significantly correlated with a prognosis of CHF. Our findings suggested that METs may be a parameter that is equalivalent to peak $\mathrm{VO}_{2}$ in terms of prognosis.

In conclusion, the present study has demonstrated that the SBP response to peak exercise, HR at the 3rd minute after exercise, and exercise tolerance (METs) obtained from routine exercise stress testing predicted mortality in a variety of heart disease patients with $\mathrm{CHF}$ irrespective of the use of $\beta$-adrenergic blocking agents. Thus, our findings may contribute to the understanding of therapeutic strategies and management of patients with $\mathrm{CHF}$.

\section{ACKNOWLEDGMENT}

We thank Emiko Shiotani for her excellent secretary assistance.

\section{REFERENCES}

1. Hunt SA, Baker DW, Chin MH, et al. ACC/AHA Guidelines for the Evaluation and Management of Chronic Heart Failure in the Adult: Executive Summary. A Report of the American College of Cardiology/American Heart Association Task Force on Practice Guidelines (Committee to Revise the 1995 Guidelines for the Evaluation and Management of Heart Failure): Developed in Collaboration With the International Society for Heart and Lung Transplantation; Endorsed by the Heart Failure Society of America. Circulation 2001; 104: 2996-3007.

2. O'Neil JO, Young JB, Pothier CE, Lauer MS. Peak oxygen consumption as a predictor of death in patients with heart failure receiving beta-blockers. Circulation 2005; 111: 2313-8.

3. Francis DP, Shamim W, Davies LC, et al. Cardiopulmonary exercise testing for prognosis in chronic heart failure: continuous and independent prognosis value from $\mathrm{VE} / \mathrm{VCO}(2)$ slope and peak VO(2). Eur Heart J 2000; 21: 154-61.

4. Guazzi M, Reina G, Tumminello G, Guazzi MD. Exercise ventilation inefficiency and cardiovascular mortality in heart failure: the critical independent prognostic value of the arterial CO2 partial pressure. Eur Heart J 2005; 26: 472-80.

5. Gauri AJ, Raxwal VK, Roux L, Fearon WF, Froelicher VF. Effects of chronotropic incompetence and beta-blocker use on the exercise treadmill test in men. Am Heart J 2001; 142: 136-41.

6. Roche F, Pichot V, Da Costa A, et al. Chronotropic incompetence response to exercise in congestive heart failure, relationship with the cardiac autonomic status. Clin Physiol 2001; 21: $335-42$.

7. Witte KK, Cleland JG, Clark AL. Chronic heart failure, chronotropic incompetence, and the effect of beta blockade. Heart 2006; 92: 481-6.

8. Robbins M, Francis G, Pashkow FJ, et al. Ventilatory and heart rate responses to exercise: better predictors of heart failure mortality than peak oxygen consumption. Circulation 1999; 100: 2411-7.

9. Lipinski MJ, Vetrovec GW, Gorelik D, Froelicher VF. The importance of heart rate recovery in patients with heart failure or left ventricular systolic dysfunction. J Card Fail 2005; 11: 62430.

10. Arena R, Guazzi M, Myers J, Peberdy MA. Prognostic value of heart rate recovery in patients with heart failure. Am Heart $\mathrm{J}$ 2006; 151: 851. e7-13.

11. Nanas S, Anastasiou-Nana M, Dimopoulos S, et al. Early heart rate recovery after exercise predicts mortality in patients with chronic heart failure. Int J Cardiol 2006; 110: 393-400.

12. Bilsel T, Terzi S, Akbulut T, Sayar N, Hobikoglu G, Yesilci- 
men $\mathrm{K}$. Abnormal heart rate recovery immediately after cardiopulmonary exercise testing in heart failure patients. Int Heart $\mathrm{J}$ 2006; 47: 431-40

13. Tanabe Y, Takahashi M, Hosaka Y, Ito M, Ito E, Suzuki K. Prolonged recovery of cardiac output after maximal exercise in patients with chronic heart failure. J Am Coll Cardiol 2000; 35: 1228-36.

14. Osada N, Chaitman BR, Miller LW, et al. Cardiopulmonary exercise testing identifies low risk patients with heart failure and severely impaired exercise capacity for heart transplantation. J Am Coll Cardiol 1998; 31: 577-82.

15. Fagard R, Pardaens K, Vanhaecke J. Prognostic significance of exercise versus resting blood pressure in patients with chronic heart failure. J Hypertens 1999; 17: 1977-81.

16. Imai K, Sato H, Hori M, et al. Vagally mediated heart rate recovery after exercise is accelerated in athletes but blunted in patients with chronic heart failure. J Am Coll Cardiol 1994; 24 : 1529-35. 